\title{
Estradiol-Induced MMP-9 Expression via PELP1-Mediated Membrane-Initiated Signaling in ERa-Positive Breast Cancer Cells
}

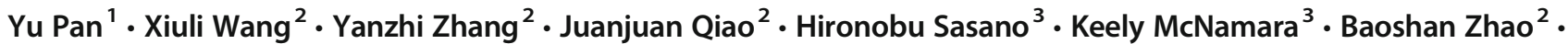 Dongmei Zhang ${ }^{2} \cdot$ Yuhua Fan ${ }^{2} \cdot$ Lili Liu $^{2} \cdot$ Xueling Jia ${ }^{2} \cdot$ Ming Liu $^{4} \cdot$ Sihang Song ${ }^{5} \cdot$ Lin Wang $^{2}$ (D)}

Received: 15 January 2020 / Accepted: 27 January 2020 / Published online: 10 February 2020

(C) Springer Science+Business Media, LLC, part of Springer Nature 2020

\begin{abstract}
Proline-, glutamic acid-, leucine-rich protein 1 (PELP1) is a novel estrogen receptor (ER) coregulator, demonstrated distinctive characters from other ER $\alpha$ coregulators, and has been suggested to be involved in metastasis of several cancers. In ER $\alpha$-positive breast cancer, PELP1 overexpression enhanced ruffles and filopodium-like structure stimulated by estradiol $\left(\mathrm{E}_{2}\right)$ through extranuclear cell signaling transduction hereby increased cell motility. However, whether PELP1 is also involved in extracellular matrix remodeling of ER $\alpha$-positive breast cancer cells is still unknown. In this study, we investigated the role of PELP1 in $\mathrm{E}_{2^{-}}$ induced MMP-9 expression and the underlined mechanism. The results demonstrated the following: $\mathrm{E}_{2}$-induced ER $\alpha$-positive MCF-7 breast cancer cell MMP-9 mRNA and protein expression in a rapid response and concentration-dependent manner. Knocked down PELP1 significantly suppressed $E_{2}$-induced MMP-9 expression. $E_{2}$-bovine serum albumin (BSA), a large molecular membrane-impenetrable conjugate of $\mathrm{E}_{2}$, can also upregulate MMP-9 protein expression in MCF-7, and the action of $\mathrm{E}_{2}$-BSA can be abolished by PI3K inhibitor LY294002; treating MCF-7 simultaneously with PELP1-shRNA and LY294002 did not show synergetic inhibitory effect on $\mathrm{E}_{2}$-BSA-induced MMP-9 expression. Our results indicated that estrogen-induced MMP-9 expression in ER-positive breast cancer cells may be through PELP1-mediated PI3K/Akt signaling pathway.
\end{abstract}

Keywords Proline-, glutamic acid-, leucine-rich protein $1 \cdot$ Matrix metalloproteinase- $9 \cdot$ Estrogen $\cdot$ Breast cancer

\section{Introduction}

Breast cancer is the most commonly diagnosed cancer and the leading cause of cancer death among women around the

Yu Pan and Xiuli Wang contributed equally to this work.

Lin Wang

wangl_cmu@126.com

1 Department of Anatomy, College of Basic Medical Sciences, Harbin Medical University-Daqing, Daqing 163319, Heilongjiang, China

2 Department of Pathology and Pathophysiology, College of Basic Medical Sciences, Harbin Medical University-Daqing, Daqing 163319, Heilongjiang, China

3 Department of Pathology, Tohoku University School of Medicine of Medicine, Sendai, Miyagi 3600107, Japan

4 Department of Pathology, the Fifth Affiliated Hospital of Harbin Medical University, Daqing 163319, Heilongiiang, China

5 Department of Histology and Embryology, College of Basic Medical Sciences, Harbin Medical University-Daqing,

Daqing 163319, Heilongjiang, China world, many of whom died from metastatic diseases [1]. The invasion and metastasis of breast cancer cells is a multi-step process, including at least three key events: (i) the enhancement of tumor cell migration ability; (ii) the damage of intercellular adhesion; (iii) the remodeling of extracellular matrix [2]. In respect of extracellular matrix remodeling, matrix metalloproteinases (MMPs) are the main functional executors based on their zinc-dependent endopeptidase activity [3]. Except for dominating the degradation of the basement membrane, MMPs are also involved in the modification of cancer cell adhesion molecules, activation of powerful cytokines, induction of epithelial-mesenchymal transition (EMT), and stimulation of tumor cell invasion [4]. In breast cancer, MMP-2 and MMP-9, especially MMP-9, play the most important role in the invasion and metastasis of tumor cells $[5,6]$.

About $70 \%$ of breast cancer cells are estrogen-dependent, in which estrogen receptors (ERs) were expressed, and the biological behaviors such as occurrence, development, metastasis, and drug resistance of the cancer cells were regulated by estrogen [7]. MMPs could be produced by tumor cells themselves in response to extracellular stimulatory interactions, 
and accumulated evidence demonstrated that the expression and activity of MMP-9 in ER-positive breast cancer cells would be regulated by estrogen or anti-estrogen reagents [8-13].

The full-length ER $\alpha$ or ER $\beta$ estrogen receptors can mediate both nuclear-initiated genomic and membrane-initiated non-genomic signaling pathway of estrogen [14] [15]. The switch between the two pathways was mainly controlled by ER coregulators. Compared with the ER itself, ER coregulators may play a more important role in regulating the biological function of estrogen. Therefore, the dysregulated expression of ER coregulators may provide breast cancer cells more advantages in the initiation, progression, metastasis, and therapeutic resistance [16].

Proline-, glutamic acid-, leucine-rich protein 1 (PELP1) is a novel ER coregulator. In the ER signaling pathway, PELP1 serves as a scaffolding protein that couples various signaling complexes with ER and participates in both genomic and nongenomic functions $[17,18]$. Overexpression of PELP1 induced malignant transformation of normal cells, accelerated cell cycle progress, promoted tumor cell proliferation, and enhanced migration and invasion of tumor cells, which supported a proto-oncogenic property of PELP1 [19]. Several recent studies have demonstrated that PELP1 was involved in EMT of both ER-negative and ER-positive breast cancer cells [20-22]. In ER-negative cells, PELP directly upregulate transcription of MMP-9 and promote cell proliferation, migration, and metastasis independent of estradiol $\left(\mathrm{E}_{2}\right)$ stimulation [22]; in ER-positive cell line, Velamudi et al. reported PELP1 overexpression enhanced $\mathrm{E}_{2}$ stimulated ruffles and filopodium-like structure through activating the membraneinitiated non-genomic signaling pathway hereby increased cell motility [20]. However, whether PELP1 is also involved in estrogen-induced expression of MMP-9 in ER-positive breast cancer cells and its possible signaling pathway is still unknown. In this study, we investigated the role of PELP1 in $\mathrm{E}_{2}$-induced MMP-9 expression and the underlined mechanism(s) in ER-positive MCF-7 breast cancer cells, to provide new insight on estrogen-induced metastasis of hormonedependent breast cancer cells.

\section{Material and Methods}

\section{Cell Culture and Reagents}

MCF-7 human breast cancer cell line was purchased from the Cell Bank of the Chinese Academy of Sciences (catalog \# $\mathrm{TCHu}$ 74), cell line authenticity was verified with STR analysis, and mycoplasma was tested by the supplier before the cell was supplied. $\mathrm{E}_{2}$ (catalog \# E2257), $\mathrm{E}_{2}$-bovine serum albumin (BSA) (catalog \# E6507), and the PI3K inhibitor LY-294002 (catalog \#440202-5MG) were purchased from
Sigma. Antibody against MMP-9 was purchased from Abcam (monoclonal, catalog \# EP1254). The PELP1 antibody was purchased from Bethyl Laboratories (polyclonal, catalog \# A300-180A). The antibody against Akt ${ }^{\mathrm{pan}}$ (monoclonal, catalog \# 4685) or Phospho-Akt Ser473 (monoclonal, catalog \# 4058) was purchased from Cell Signaling Technology.

\section{Generation of PELP1-shRNA Model Cells}

MCF-7 stable expressing PELP1-shRNA (MCF-7 ${ }^{\text {PELP1- }}$ shRNA) was generated with the MISSION Lentiviral Transduction Particles (catalog \# SHCLNV, Sigma) and selected with Puromycin $(1 \mu \mathrm{g} / \mathrm{mL})$. PELP1-shRNA design was based on the GenBank accession number NM_014389. The PELP1-specific Sure Silencing shRNA plasmids (catalog \# TRCN0000159617, TRCN0000159673, TRCN0000159883, and TRCN0000159193) and non-specific control shRNA vector (NC, catalog \# SHC002V) were purchased from Sigma, the assay was performed according to the technical bulletin of the product, and pooled clones were used for subsequent experiments.

\section{Treatment of Cell Lines}

MCF-7 cell line was maintained in DMEM medium (Gibco) supplemented with $10 \%$ heat-inactivated fetal bovine serum (FBS, Biological Industries) at $37^{\circ} \mathrm{C}$ with $5 \% \mathrm{CO}_{2}$. Before $\mathrm{E}_{2}$ or $\mathrm{E}_{2}$-BSA treatment, the medium was changed to complete phenol red-free DMEM (Gibco) supplemented with 5\% charcoal-stripped FBS (Hyclone). $\mathrm{E}_{2}$ was dissolved and stocked in ethanol at a concentration of $1 \mathrm{mM}$ and was added into the culture medium when used for treatment of the cells; the final concentration of ethanol in medium was no more than $1 / 10000$. $\mathrm{E}_{2}-\mathrm{BSA}$ was dissolved in phosphate-buffered saline (PBS), and free $\mathrm{E}_{2}$ was removed by filtration using the technique described by Stevis et al. [23]. The filtered solution was added into the culture medium when used for treatment of the cells; $\mathrm{E}_{2}$ concentrations were calculated with $10 \mathrm{~mol} \mathrm{E}_{2}$ per mol bovine serum albumin (BSA) according to manufacturer's specifications.

\section{RT-PCR}

Total RNA was extracted using TRIzol reagent (Invitrogen Life Technologies). First-strand cDNA was synthesized with M-MLV transcriptase (Promega) and oligo dT. RT-qPCR was conducted using SYBR Green PCR master mix (Takara Bio, Inc.). The primers used for amplifying MMP-9 cDNA were designed as follows: 5'-ATTTCTGCCAGGACCGCTTC TACT-3' (forward), 5'-CAGTTTGTATCCGGCAAACT GGCT-3 (reverse); primers for $\beta$-actin were $5^{\prime}$-GGAC TTCGAGCAAGAGATGG-3' (forward) and 5'-ACAT 
CTGCTGGAAGGT-GGAC-3' (reverse). All samples were performed in triplicates. Values were normalized by $\beta$-actin and calculated with $\Delta \Delta \mathrm{CT}$ method.

\section{Western Blot}

Proteins were extracted with RIPA lysis buffer. The proteins were separated by sodium dodecyl sulfate polyacrylamide gel electrophoresis (SDS-PAGE) and then transferred onto a nitrocellulose blotting membrane. The membrane was blocked in 5\% skimmed milk, then washed three times with Trisbuffered saline with $0.05 \%$ Tween 20 (TBS-T), and probed with PELP1 (1/500), MMP-9 (1/2000), Akt ${ }^{\text {pan }}(1 / 1000)$, Phospho-Akt Ser473 (1/1000), or $\beta$-actin (Santa Cruz, $1 / 5000$ ) primary antibodies overnight at $4{ }^{\circ} \mathrm{C}$. The samples were incubated with the appropriate secondary antibodies for $1 \mathrm{~h}$. The assays were performed in triplicates. Quantification of the bands was analyzed with the NIH Image J program.

\section{Immunohistochemistry}

A total of 109 formalin-fixed, paraffin-embedded ER $\alpha$ positive breast cancer tissue blocks collected from the Fifth Affiliated Hospital of Harbin Medical University were used for detecting the protein expression of MMP-9 in this study. The tissue samples had been implored in our previous study for investigating the PELP1 expression pattern in Chinese women with primary breast cancer [24]. None of the patients underwent chemotherapy, radiotherapy, or endocrine therapy before surgical excision. The protocol of this study was approved by the institutional review board of Harbin Medical University-Daqing; written informed consent was obtained from all participants. Immunostaining of PELP1was performed as previously reported [24]. For MMP-9 immunostaining, sample blocks were sliced to $4-\mu \mathrm{m}$-thick serial section, deparaffinized with xylene, and rehydrated on alcohol gradients. Endogenous peroxidase was blocked with $3 \%$ hydrogen peroxide in methanol for 30 min before antigen retrieval with microwave. The sliders were incubated with diluted antibody against MMP-9 $(1 / 200)$ under $4{ }^{\circ} \mathrm{C}$ overnight. Real Envision Detection system (DAKO, Denmark) was used in accordance with the manufacturer's instruction. Sections were visualized with the chromogen DAB and counterstained with hematoxylin. Negative controls were performed by omitting the primary antibody and substituting with antibody dilution buffer (DAKO, Denmark). The evaluation of immunoreactivity was performed independently by two of the authors (BS.Z. and M.L.). $H$-score was used to assess immunoreactivity of PELP1 as previously described [25]. The evaluation of MMP-9 immunoreactivity followed the method of $\mathrm{Xu}$ et al. [26].

\section{Statistical Analysis}

Data from the in vitro studies were expressed as mean \pm S.E.M. of three independent experiments and statistical differences were analyzed with one-way ANOVA followed by the Tukey-Kramer test. The correlation between PELP1 and MMP-9 expression in breast cancer tissues samples was analyzed with Spearman correlation test. A value of $P<0.05$ was considered statistically significant.

\section{Results}

\section{$E_{2}$-Induced mRNA and Protein Expression of MMP-9 in MCF-7 Demonstrated a Rapid Response and Concentration-Dependent Pattern}

In order to explore the effects of $\mathrm{E}_{2}$ on the expression of MMP9 in ER $\alpha$-positive breast cancer cells, real-time PCR and Western blot were performed. Both the mRNA and protein expression of MMP-9 in ER $\alpha$-positive MCF-7 breast cancer cells demonstrated a rapid increase, which appeared at $10 \mathrm{~min}$ $(1 / 6 \mathrm{~h})$ and reached peak at $1 \mathrm{~h}$ after treated with10nM $\mathrm{E}_{2}$ (Fig. 1a, b). To examining the concentration-effect relation of $\mathrm{E}_{2}$-induced MMP-9 expression, MCF-7 cells were treated with 1 100 $\mathrm{nM} \mathrm{E}_{2}$ for $1 \mathrm{~h}$; MMP-9 mRNA (Fig. 1c) and protein (Fig. 1d) showed $E_{2}$ concentration-dependent increase.

\section{Membrane-Impenetrable $E_{2}-B S A-I n d u c e d ~ m R N A$ and Protein Expression of MMP-9 in MCF-7 with a Pattern Similar to $E_{2}$}

$\mathrm{E}_{2}$-BSA, a large molecular conjugate of $\mathrm{E}_{2}$ with $\mathrm{BSA}$, was reported to be membrane-impenetrable and be able to activate the membrane-initiated estrogen signaling. Since the $\mathrm{E}_{2}-\mathrm{BSA}$ (catalog \# E6507, Sigma) used in this study was labeled with FITC, we firstly examined the membrane penetrability of $E_{2}-$ BSA with fluorescence microscopy. As shown in Fig. 2a, after adding $10 \mathrm{nM} \mathrm{E}_{2}$-BSA (molar concentration of $\mathrm{E}_{2}$, equal to concentration of $1 \mathrm{nM} \mathrm{BSA}$ according to manufacturer's specifications) into the culture medium, the green fluorescence could only been observed around the outline of alive cells. However, the green fluorescence could been observed in the whole cell when the cells were treated with Triton X100. This result suggested that $\mathrm{E}_{2}$-BSA could not penetrate the membrane when the cells were alive. Subsequently, we detect an MMP-9 expression in MCF-7 treated with $10 \mathrm{nM} \mathrm{E}_{2}$-BSA in different time periods. Similar to that induced by $\mathrm{E}_{2}$, both mRNA and protein expressions of MMP-9 demonstrated a significant increase at $30 \mathrm{~min}$ and reached peak at $1 \mathrm{~h}$ after the stimulation with $\mathrm{E}_{2}$-BSA (Fig. 2b, c). Furthermore, we compared the effect of $\mathrm{E}_{2}$-BSA and $\mathrm{E}_{2}$ at the concentration respectively of $10 \mathrm{nM}$ and $100 \mathrm{nM}$ on promoting mRNA and 


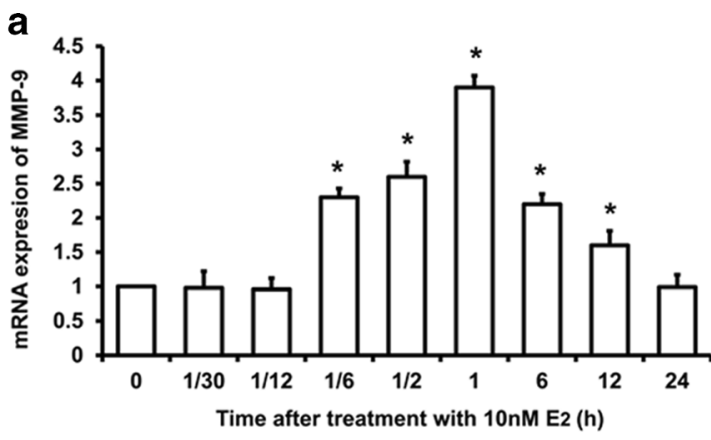

b
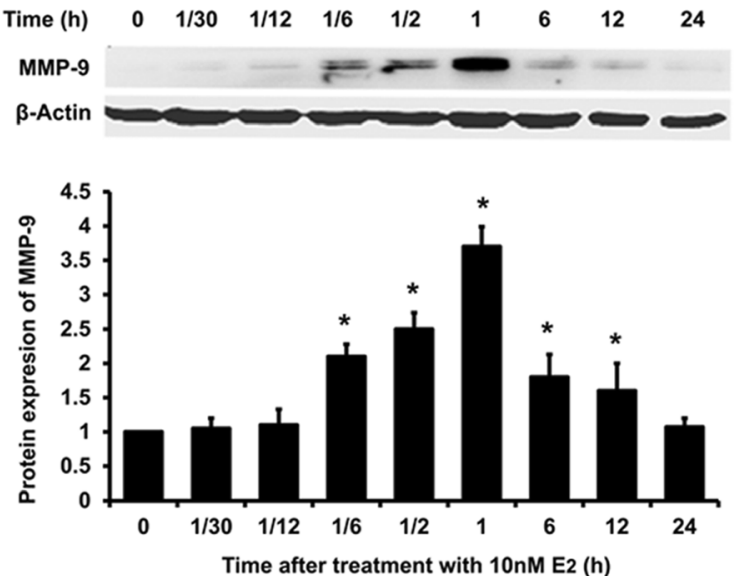

Fig. $1 \mathrm{E}_{2}$-induced MMP-9 expression in MCF-7 breast cancer cells. $\mathrm{ER} \alpha$-positive MFC-7 breast cancer cell was treated with $10 \mathrm{nM} \mathrm{E}_{2}$ for different time periods; mRNA (a) and protein expression (b) of MMP-9 were detected respectively by real-time PCR and Western blot. For investigating the concentration-response relation between $\mathrm{E}_{2}$ treatment

protein expression of MMP-9. It is demonstrated that $\mathrm{E}_{2}-\mathrm{BSA}$ concentration dependently increased mRNA and protein expression of MMP-9 in MCF-7 cells (Fig. 2d, e). Moreover, the expression of MMP-9 mRNA and protein did not show significant difference between equal molar concentration of $E_{2-}$ BSA and $\mathrm{E}_{2}$ (Fig. 2d, e). This result indicated the membraneinitiated estrogen signaling conducted by $\mathrm{E}_{2}$-BSA was enough to exert entire regulatory function of estradiol on MMP-9 expression in ER $\alpha$-positive breast cancer cells.

\section{Knocking Down PELP1 Significantly Attenuated $E_{2}$ and $E_{2}-B S A-I n d u c e d$ MMP-9 Expression in MCF-7}

It is reported PELP1 plays a critical role in the membraneinitiated estrogen signaling. We thereby established a PELP1 knocking down cell model-based MCF-7 by transfecting PELP1-shRNA. Western blot demonstrated that transfection of catalog \# TRCN0000159883 PELP1-specific Sure Silencing shRNA plasmids obtained the optimal knocking down efficiency (data not shown), which demonstrated a 0.26-fold protein expression of PELP1 compared with that in NC transfected cells (Fig. 3a). Knocking down PELP1

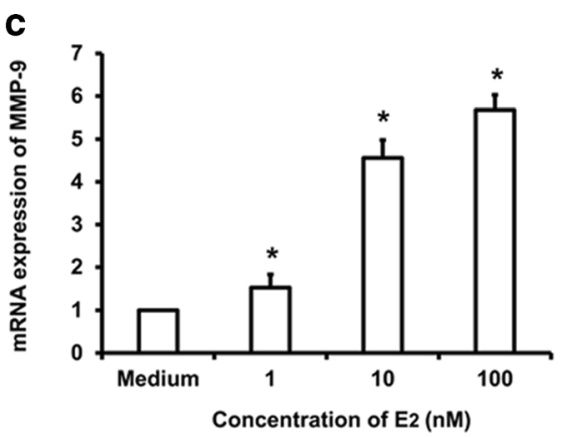

d 92KD 42KD
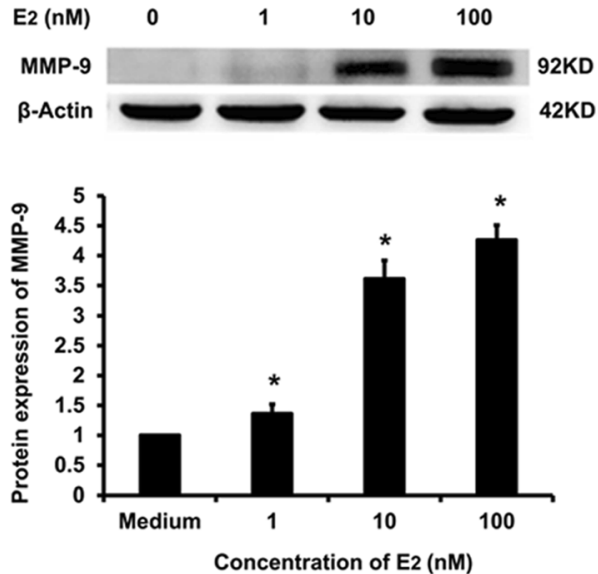

and expression of MMP-9, MCF-7 was treated with 1 100 nM E $\mathrm{E}_{2}$ for $1 \mathrm{~h}$, and mRNA (c) and protein expression (d) of MMP-9 were detected respectively by real-time PCR and Western blot. All presented figures are representative data from at least three independent experiments. $* P<0.05$ compared with $0 \mathrm{~h}$ of $\mathrm{E}_{2}$ treatment $(\mathbf{a}, \mathbf{b})$ or medium control $(\mathbf{c}, \mathbf{d})$

significantly attenuated the mRNA and protein expression of MMP-9 in MCF-7 induced either by $E_{2}$ or $E_{2}$-BSA (Fig. 3b, c). The result confirmed the critical role of PELP1 in estradiolinduced expression of MMP-9 in ER $\alpha$-positive breast cancer cells via the membrane-initiated estrogen signaling.

\section{PI3K/Akt Pathway Downstream PELP1 Was Involved in $\mathrm{E}_{2}$-BSA-Induced MMP-9 Expression in MCF-7}

IP3K/Akt pathway had been reported to be involved in the downstream of PELP1-mediated membrane-initiated estrogen signaling. We further investigated whether inhibiting activity of PI3K could influence $\mathrm{E}_{2}$-BSA-induced expression of MMP-9 in MCF-7 using the PI3K inhibitor LY-294002 alone or in combination with PELP1-shRNA. The mRNA expression of MMP-9 was detected with RT-PCR, and Western blot was performed to examine MMP-9 and PELP1 protein expression of and phosphorylation of Ser478 residue of Akt (the main downstream substrate of PI3K). It is revealed that either LY-294002 or PELP1-shRNA alone suppressed phosphorylation of Akt and impeded both mRNA and protein expression of MMP-9 induced by $\mathrm{E}_{2}-\mathrm{BSA}$ in MCF-7. Treated 


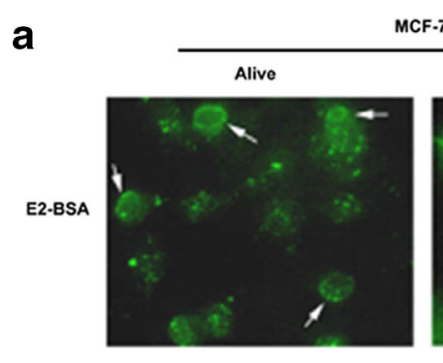

MCF-7

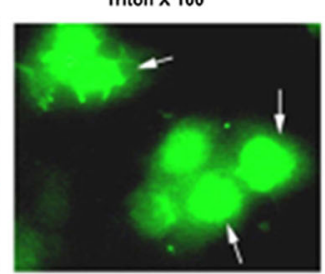

d
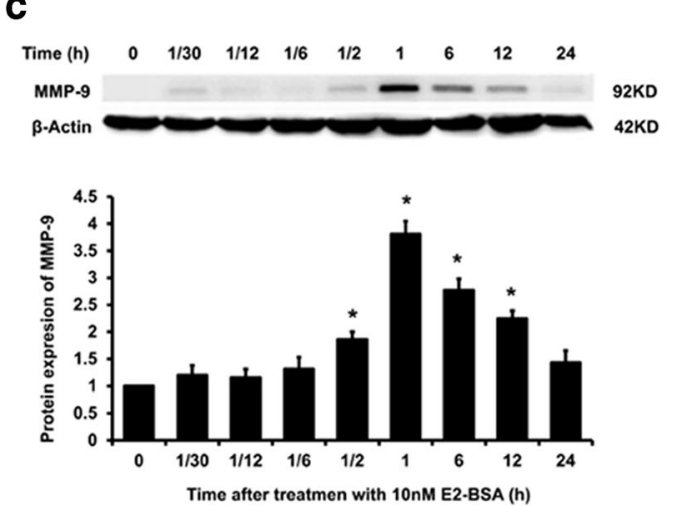

Fig. 2 Membrane-impenetrable $\mathrm{E}_{2}$-BSA-induced mRNA and protein expression of MMP-9 in MCF-7. Fluorescent photography showed that FITC-labeled $\mathrm{E}_{2}$-BSA did not puncture the membranes of MCF-7 breast cancer cells (a). MFC-7 was treated with $10 \mathrm{nM} \mathrm{E}_{2}$-BSA for different time periods; the mRNA (b) and protein expression (c) of MMP-9 were detected respectively by real-time PCR and Western blot. Subsequently, MFC-7 was treated with $\mathrm{E}_{2}-\mathrm{BSA}$ and $\mathrm{E}_{2}$ at the concentration respectively

cells with LY-294002 in combination with PELP1-shRNA totally abolished $\mathrm{E}_{2}$-BSA-induced increment of mRNA and protein expression of MMP-9 (Fig. 4). This result indicated IP3K/Akt pathway may play an important role downstream PELP1 in $\mathrm{E}_{2}$-BSA-induced MMP-9 expression in ER $\alpha$ positive breast cancer cells. b

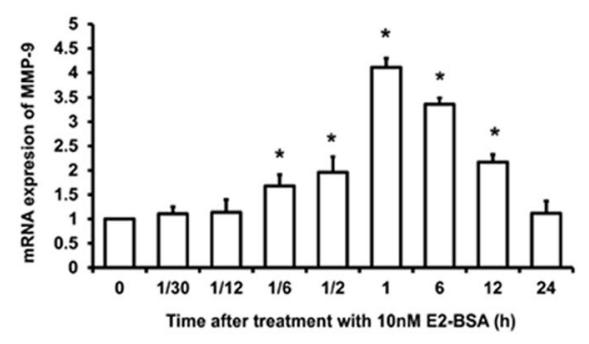

e
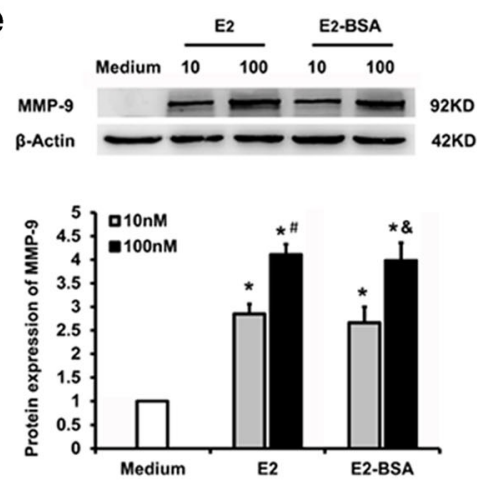

of $10 \mathrm{nM}$ and $100 \mathrm{nM}$; the mRNA (d) and protein expression (e) of MMP-9 were detected respectively by real-time PCR and Western blot. All presented figures are representative data from at least three independent experiments. $* P<0.05$ compared with $0 \mathrm{~h}$ of $\mathrm{E}_{2}$-BSA treatment $(\mathbf{b}, \mathbf{c})$ or medium control $(\mathbf{d}, \mathbf{e}) ;{ }^{\#} P<0.05$ compared with that treated with $10 \mathrm{nM} \mathrm{E}_{2} ;{ }^{\&} P<0.05$ compared with that treated with $10 \mathrm{nM}$ $\mathrm{E}_{2}$-BSA

\section{The Expression of MMP-9 Was Positively Correlated with the Expression of PELP1 in ERa-Positive Breast Cancer Tissues}

In order to verify the role of PELP1 in estradiol-induced expression of MMP-9 in primary breast cancer, we detected

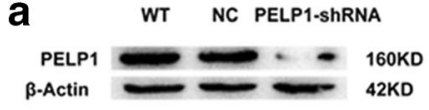

b
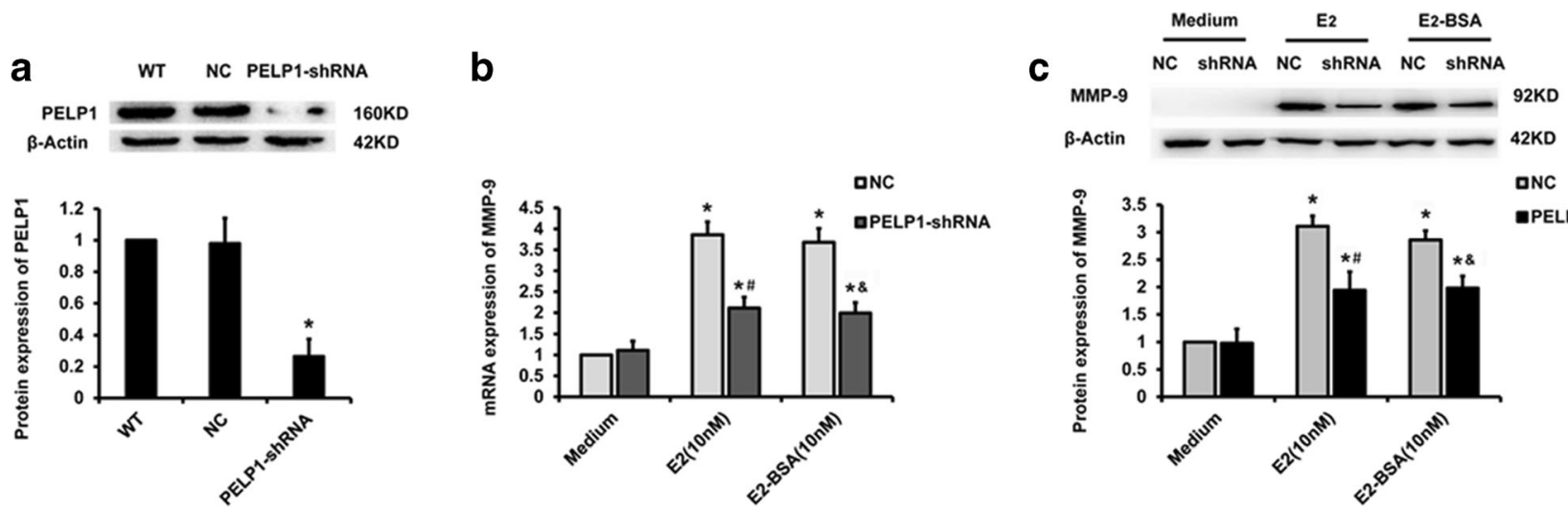

aNC

Fig. 3 The influence of knocking down PELP1 on $\mathrm{E}_{2}$ and $\mathrm{E}_{2}-\mathrm{BSA}$ induced MMP-9 expression in MCF-7. Either PELP1-shRNA or nonspecific control (NC) plasmid was transfected into MCF-7, after selected with puromycin, the protein expression of PELP1 was examined by Western blot (a). MFC-7 transfected with NC or PELP1shRNA were treated with $10 \mathrm{nM}$ of either $\mathrm{E}_{2}$ or $\mathrm{E}_{2}$-BSA; the mRNA (b)

and protein expression (c) of MMP-9 were detected respectively by realtime PCR and Western blot. All presented figures are representative data from at least three independent experiments. $* P<0.05$ compared with $\mathrm{NC}$ and medium treated control; ${ }^{\#} P<0.05$ compared with that $\mathrm{NC}$ treated with $10 \mathrm{nM} \mathrm{E}_{2} ;{ }^{\&} P<0.05$ compared with that $\mathrm{NC}$ treated with $10 \mathrm{nM} \mathrm{E}_{2}$-BSA 
the expression of MMP- 9 and PELP1 in 109 cases of ER $\alpha$ positive breast cancer tissues by immunohistochemistry (IHC) (Fig. 5). MMP-9 immunostaining mainly located in plasma of tumor cells and/or extrocellular matrix around tumor cells, and PELP1 immunostaining was localized to the nuclei of tumor cells. Among 109 cases of tissue samples, 27 cases demonstrated negative MMP-9 immunostaining, 25 cases demonstrated weak MMP-9 immunostaining, 28 cases demonstrated moderate MMP-9 immunostaining, and 29 cases demonstrated strong MMP-9 immunostaining; as for PELP1, 19 cases were assessed negative, 44 cases were assessed moderate, and 46 cases were assessed strong immunostaining according to the record of our previous study [24]. Spearman correlation test showed that the protein expression of MMP-9 was positively correlated with the expression of PELP1 in ER $\alpha$-positive breast cancer tissues with an $r$ value of 0.293 and a $P$ value of 0.002 (Table 1). This result further confirmed the important role of PELP1 on estrogen-regulated expression of MMP-9 in ER $\alpha$-positive breast cancer cells.
Fig. 4 The influence of inhibiting PI3K/Akt pathway on $\mathrm{E}_{2}$-BSAinduced MMP-9 expression in MCF-7. MCF-7 was treated with PI3K inhibitor LY-294002 alone or in combination with PELP1shRNA before being stimulated with $\mathrm{E}_{2}-\mathrm{BSA}$; real-time PCR was performed to examine mRNA expression of MMP-9 (a) and Western blot were performed to examine the protein expression of MMP-9, PELP1, and phosphorylation of $\mathrm{Ser}^{478}$ residue of Akt (b). All presented figures are representative data from at least three independent experiments. $* P<0.05$ compared with $\mathrm{NC}$ and medium treated control; ${ }^{\#} P<0.05$ compared with that $\mathrm{NC}$ treated with $10 \mathrm{nM} \mathrm{E}_{2}-$ BSA

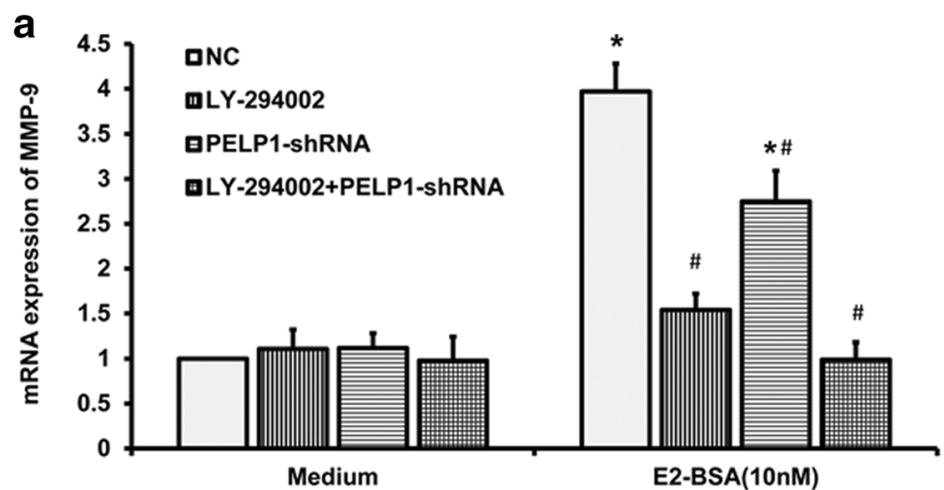

b
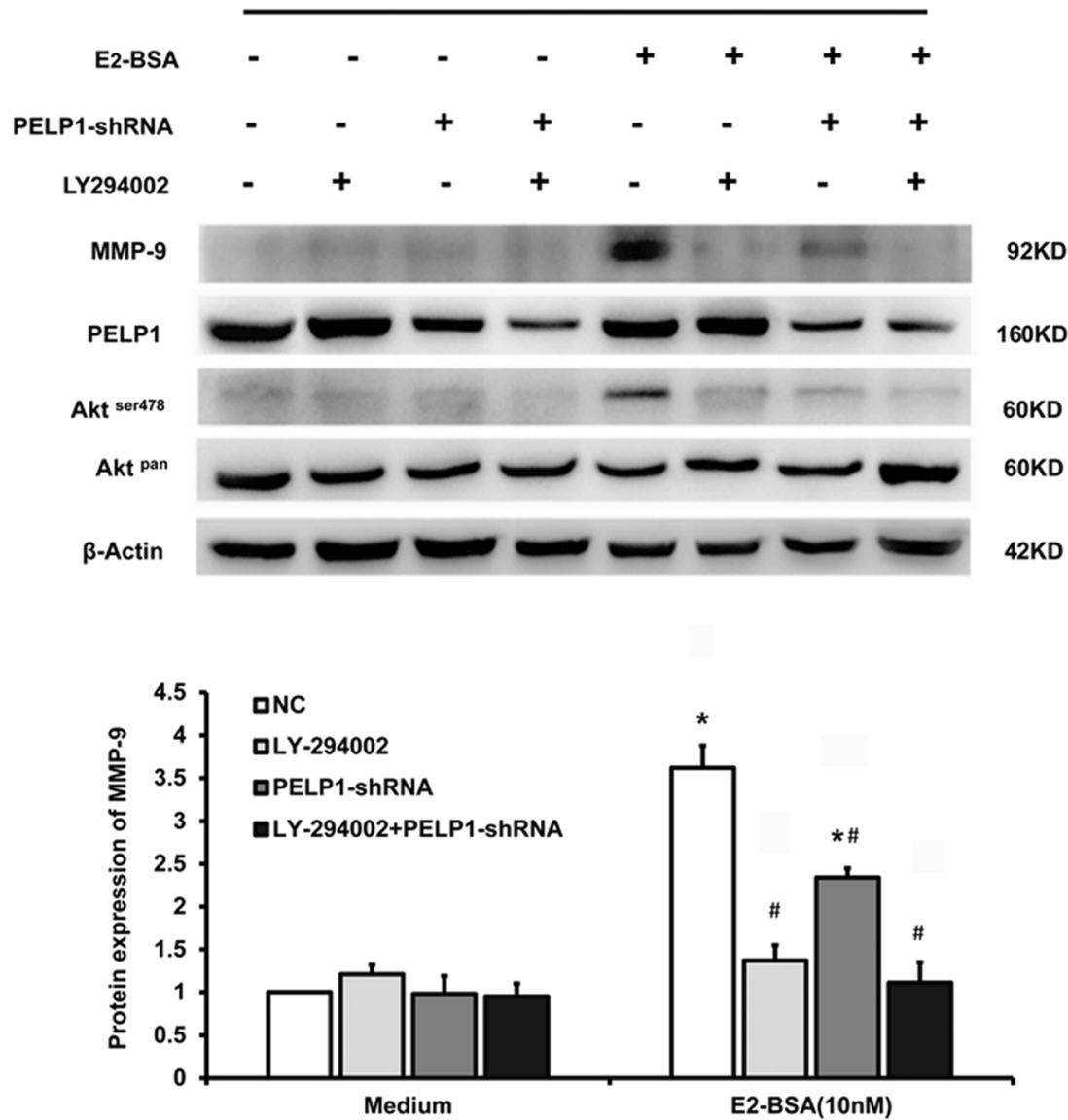

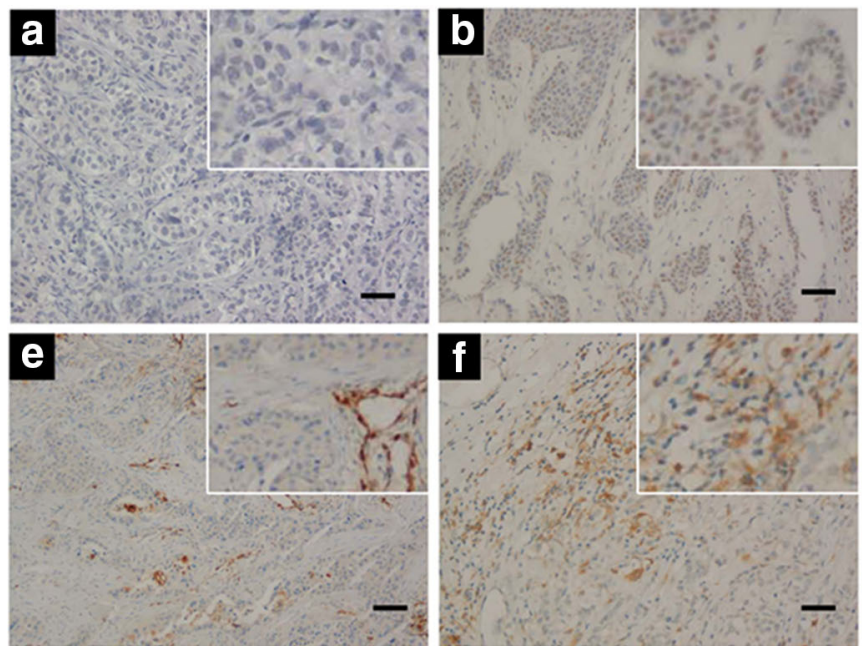

Fig. 5 Immunostaining of PELP1 and MMP-9 in ER $\alpha$-positive breast cancer tissues. a Negative immunostaining of PELP1 in breast cancer tissues. b Moderate immunostaining of PELP1 in breast cancer tissues. c Strong immunostaining of PELP1 in breast cancer tissues. d Negative immunostaining of MMP-9 in breast cancer tissues. e Weak

\section{Discussion}

Although previous studies have shown that PELP1 is involved in the invasion and metastasis of both ER-positive and ERnegative breast cancer, little is known about the role and downstream pathway of PELP1 in estrogen-induced MMP-9 expression in ER-positive breast cancer cells. Our study provided three new insights into this issue: first, estradiol stimulated rapid expression of MMP-9 in ER-positive breast cancer cells via membrane-initiated signaling; second, PELP1 played a critical role in estradiol-induced expression of MMP-9 of ER-positive breast cancer cells; third, PI3K/Akt kinase cascade acted as the downstream pathway of PELP1 in estradiolinduced MMP-9 expression of ER-positive breast cancer cells.

Though numerous reports had discussed the correlation between estrogen signal and the expression and activity of MMPs, the confliction on the direction of estrogen- or antiestrogen agent-regulated expression of MMP-9 still exists [8, $10,12,13,27-30]$. These discrepancies may be partially due to the difference in target cell types stimulated by estrogen. However, even in the same cell type, for example, in MCF-7 breast cancer cells, the regulatory effect of estrogen on the
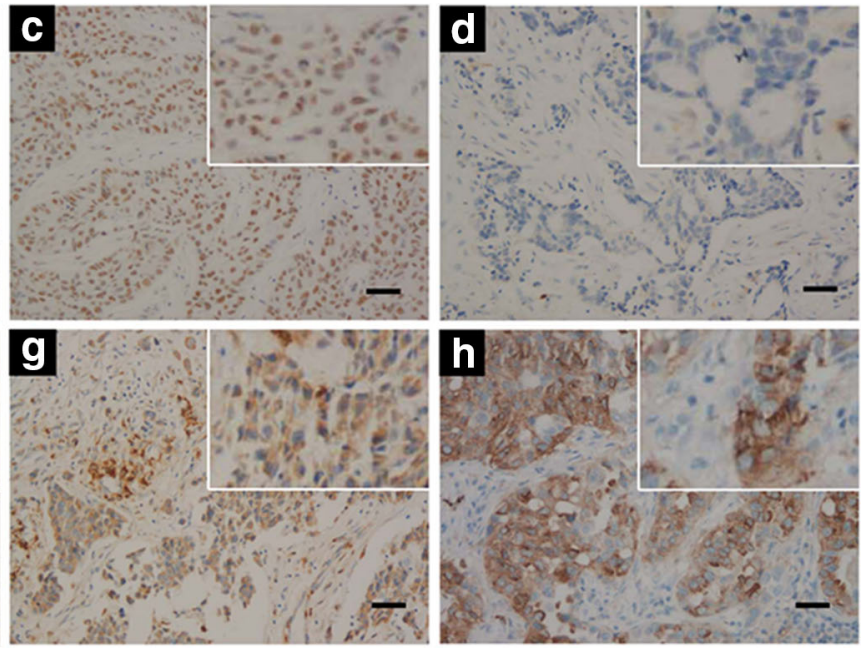

immunostaining of MMP-9 in breast cancer tissues. f Moderate immunostaining of MMP-9 in breast cancer tissues. g Strong immunostaining of PELP1 in breast cancer tissues. $\mathbf{h}$ Positive control of MMP-9 immunostaining (lung adenocarcinoma). Bar $=20 \mathrm{um}$

expression or activity of MMP-9 is still controversial. Some studies have reported that estradiol upregulated the expression and activity of MMP-9 in MCF-7 [8, 10, 27]; on the contrary, there is also a considerable number of studies reporting that estradiol downregulated MMP-9 expression in MCF-7 breast cancer cells [29, 31, 32]. A possible reason for these conflicting results may be the diversity of observation time after estradiol treatment. Notably, most observations on MMP-9 expression regulated by estrogen were more than $24 \mathrm{~h}$ after the cells were treated; few studies have paid attention to the influence of estrogen on the MMP-9 expression within minutes or hours.

In line with that reported by Razandi et al. [30] and Russo et al. [33], our study demonstrated that $17 \beta$-estradiol $\left(\mathrm{E}_{2}\right)$ induced MMP-9 mRNA and protein expression in ER $\alpha$ positive MCF-7 breast cancer cells in a rapid response and concentration-dependent manner. After being treated with 1$100 \mathrm{nM}$ of $\mathrm{E}_{2}$ for $10 \mathrm{~min}$, the mRNA and protein expression of MMP-9 in MCF-7 started to increase and reached the peak at $1 \mathrm{~h}$, then the mRNA and protein levels of MMP-9 decreased gradually and recovered to the level before $\mathrm{E}_{2}$ treatment after $24 \mathrm{~h}$. This change of MMP-9 can also be induced by membrane-impenetrable $\mathrm{E}_{2}$-BSA, with this result, suggesting
Table 1 Correlated MMP-9 expression to PELP1 in ERpositive breast cancer tissues

\begin{tabular}{|c|c|c|c|c|c|c|}
\hline \multirow{2}{*}{$\begin{array}{l}\text { Immunoactivity of } \\
\text { PELP1 }\end{array}$} & \multicolumn{4}{|c|}{ Immunoactivity of MMP-9 } & \multirow[t]{2}{*}{$r$} & \multirow[t]{2}{*}{$P$} \\
\hline & $\begin{array}{l}\text { Negative } \\
(n=27)\end{array}$ & $\begin{array}{l}\text { Weak } \\
(n=25)\end{array}$ & $\begin{array}{l}\text { Moderate } \\
(n=28)\end{array}$ & $\begin{array}{l}\text { Strong } \\
(n=29)\end{array}$ & & \\
\hline Negative $(n=19)$ & $8(42.1 \%)$ & $6(31.6 \%)$ & $2(10.5 \%)$ & $3(15.8 \%)$ & 0.293 & 0.002 \\
\hline Moderate $(n=44)$ & $12(27.3 \%)$ & $10(22.7 \%)$ & $14(31.8 \%)$ & $8(18.2 \%)$ & & \\
\hline Strong $(n=46)$ & $7(15.2 \%)$ & $9(19.6 \%)$ & $12(26.1 \%)$ & $18(39.1 \%)$ & & \\
\hline
\end{tabular}


that the rapid change of MMP-9 expression induced by $E_{2}$ in MCF-7 may be conducted by the membrane-initiated signaling pathway of estrogen. Combining our results and previous reports, we present the hypothesis that a multiple phasic pattern of estrogen-regulated expression of MMP-9 in ERpositive cells exists, which may include at least a transient regulation phase and a long-term regulation phase. The transient regulatory effect of estrogen is relatively simple, which results in the upregulation of MMP-9 expression in ERpositive cells, while the long-term regulatory effect is complex, which may be affected by certain intracellular or extracellular signal cross-talk, thereby demonstrating different regulatory results. Of course, to support this hypothesis, more comprehensive pieces of evidence will be needed.

Razandi's study described G protein-coupled estrogen receptor (GPER) as the mediator of membrane-initiated estrogen signaling for regulation of MMP-9 expression in MCF-7 [30]. However, many studies also emphasized the critical role of classical $\mathrm{ER} \alpha$ in mediating $\mathrm{E}_{2}$-regulated expression of MMP-9. Interestingly, Razandi' study was published in 2003, while it was in 2002 that PELP1 was first recognized as an estrogen receptor coregulator which plays a key role in ER $\alpha$-mediated membrane-initiated estrogen signaling [19]. Thus, PELP1 would be a promising candidate for connecting $\mathrm{ER} \alpha$ and membrane-initiated estrogen signaling in the regulation of MMP-9 expression. To our best knowledge, the role of PELP1 in $\mathrm{E}_{2}$-induced transient expression of MMP-9 was not investigated before. In the current study, we demonstrated that knocking down PELP1 with specific shRNA significantly inhibited $\mathrm{E}_{2}$-BSA-induced MMP-9 expression in MCF-7. Moreover, knocking down PELP1 could also suppress $\mathrm{E}_{2^{-}}$ BSA-induced activation of PI3K, presented as less phosphorylation of Akt. These results suggested that PELP1 plays a key role in $\mathrm{E}_{2}$-induced transient expression of MMP-9 in MCF-7 and PI3K/Akt kinase cascade may mediate the downstream signaling of PELP1.

PELP1 has no enzyme activity. Under the stimulation of estrogen, PELP1 acts as a scaffold protein to couple ER $\alpha$-cSrc-PI3K-p85 forming a multi-molecular complex, by which directly activated PI3K-p85 subunit as well as the downstream Akt signaling pathway [17]. In our study, pretreating the MCF-7 cells with PI3K inhibitor LY-294002 could also significantly suppress $\mathrm{E}_{2}$-BSA-induced expression of MMP-9 and treated the cells simultaneously with PELP1-shRNA and LY-294002 did not show synergetic inhibitory effect. This result confirmed PI3K/Akt kinase cascade as the downstream signaling pathway of PELP1-mediated MMP-9 expression in MCF-7. It is notable that both mRNA and protein levels of MMP-9 in MCF-7 rapidly changed after the cells were treated with $E_{2}$ or $E_{2}-B S A$, which suggested that certain transcriptional factor downstream PI3K/Akt kinase cascade would be necessary. Several studies have reported NFKB, as a transcriptional regulator downstream PI3K/Akt, was involved in various factors inducing expression of MMP-9 [34-36]. In this study, we did not test whether $\mathrm{NF} \kappa \mathrm{B}$ played a role in the $\mathrm{E}_{2}$-induced rapid expression of MMP-9 in MCF-7 cells; this is one of the drawbacks of the current study.

To further verify the results of the in vitro study, we detected the expression of PELP1 and MMP-9 in 104 ER $\alpha$-positive breast cancer tissues by IHC. Echoing to the results from the in vitro study, the PELP1 expression was positively correlated with the MMP-9 expression in the breast cancer tissues. It is worth noting that although the in vitro study suggested $E_{2}-$ induced MMP-9 expression via PELP1-mediated membraneinitiated signaling, we only detected exclusive nuclear localization of PELP1, without cytoplasm or membrane immunostaining of PELP1, was observed. A possible explanation for this discrepancy may lie in the antibody against PELP1 used in this study. The antibody against PELP1 used in this study (Bethyl Laboratory; cat \# IHC-00013) and most commercially available antibodies against PELP1 were raised to recognize the epitopes between residues 1000 and 1050 in the Cterminal of PELP1, which has been identified as a region for PELP1 interaction with cytoplasmic proteins, such as the p85 subunit of PI3K $[37,38]$. Thus, the epitope recognized by the antibody used in this study might be masked when PELP1 is localized in the cytoplasm and leave only nuclear immunostaining detectable by IHC.

In conclusion, we confirmed the key role of PELP1 in $\mathrm{E}_{2-}$ induced rapid expression of MMP-9 in ER-positive breast cancer cells and explored the possible signaling pathway downstream PELP1 in this study. There are still some deficiencies in the study. For example, we did not observe the secretion and activation of MMP-9 induced by estradiol in MCF-7, did not explore the downstream pathway of $\mathrm{PI} 3 \mathrm{~K} / \mathrm{Akt}$, and did not test the multiple-phasic-pattern hypothesis of estrogen-regulated MMP-9 expression in ER-positive breast cancer cells; these issues will be investigated in our future studies.

Authors' Contributions Yu Pan, Xiuli Wang, Yanzhi Zhang, Ming Liu, Lili Liu, Sihang Song, Xueling Jia, and Baoshan Zhao performed the research; Lin Wang, Keely M. McNamara, Hironobu Sasano, and Yuhua Fan designed the research study; Juanjuan Qiao and Dongmei Zhang analyzed the data; Yu Pan and Lin Wang wrote the paper. All authors read and approved the final manuscript.

Funding information This work was supported by the Fundamental Research Funds for the Provincial Universities (Grant No. JFXN201906 and JFXN201910), the Scientific Research Fund of Harbin Medical University-Daqing (Grant No. DQ2013-1), and the Postgraduate Tutor Foundation of Harbin Medical University-Daqing (Grant No. DSJJ2015003).

\section{Compliance with Ethical Standards}

Conflict of Interest The authors declare that we have no conflict of interest. 
Ethical Approval All procedures performed in studies involving human participants were in accordance with the ethical standards of the institutional review board of Harbin Medical University-Daqing and with the 1964 Helsinki declaration and its later amendments or comparable ethical standards.

\section{References}

1. Bray F, Ferlay J, Soerjomataram I, Siegel RL, Torre LA, Jemal A (2018) Global cancer statistics 2018: GLOBOCAN estimates of incidence and mortality worldwide for 36 cancers in 185 countries. CA Cancer J Clin 68:394-424

2. Valastyan S, Weinberg RA (2011) Tumor metastasis: molecular insights and evolving paradigms. Cell 147:275-292

3. Kleiner DE, Stetler-Stevenson WG (1999) Matrix metalloproteinases and metastasis. Cancer Chemother Pharmacol 43(Suppl):S42S51

4. Cui N, Hu M, Khalil RA (2017) Biochemical and biological attributes of matrix metalloproteinases. Prog Mol Biol Transl Sci 147: $1-73$

5. Holanda AO, Oliveira AR, Cruz KJ, Severo JS, Morais JB, Silva BB, Marreiro DD (2017) Zinc and metalloproteinases 2 and 9: what is their relation with breast cancer? Rev Assoc Med Bras(1992) 63: 78-84

6. Ren F, Tang R, Zhang X, Madushi WM, Luo D, Dang Y, Li Z, Wei $\mathrm{K}$, Chen G (2015) Overexpression of MMP family members functions as prognostic biomarker for breast cancer patients: a systematic review and meta-analysis. PLoS One 10:e0135544

7. Lin SX, Chen J, Mazumdar M, Poirier D, Wang C, Azzi A, Zhou M (2010) Molecular therapy of breast cancer: progress and future directions. Nat Rev Endocrinol 6:485-493

8. Abdallah MA, Abdullah HI, Kang S, Taylor DD, Nakajima ST, Gercel-Taylor C (2007) Effects of the components of hormone therapy on matrix metalloproteinases in breast-cancer cells: an in vitro study. Fertil Steril 87:978-981

9. Bendrik C, Robertson J, Gauldie J, Dabrosin C (2008) Gene transfer of matrix metalloproteinase- 9 induces tumor regression of breast cancer in vivo. Cancer Res 68:3405-3412

10. Kousidou O, Berdiaki A, Kletsas D, Zafiropoulos A, Theocharis AD, Tzanakakis GN, Karamanos NK (2008) Estradiol-estrogen receptor: a key interplay of the expression of syndecan- 2 and metalloproteinase-9 in breast cancer cells. Mol Oncol 2:223-232

11. Mitropoulou TN, Tzanakakis GN, Kletsas D, Kalofonos HP, Karamanos NK (2003) Letrozole as a potent inhibitor of cell proliferation and expression of metalloproteinases (MMP-2 and MMP9) by human epithelial breast cancer cells. Int J Cancer 104:155160

12. Nilsson UW, Garvin S, Dabrosin C (2007) MMP-2 and MMP-9 activity is regulated by estradiol and tamoxifen in cultured human breast cancer cells. Breast Cancer Res Treat 102:253-261

13. Wolczynski S, Surazynski A, Swiatecka J, Palka J (2001) Estrogenic and antiestrogenic effects of raloxifene on collagen metabolism in breast cancer MCF-7 cells. Gynecol Endocrinol 15: 225-233

14. Arnal JF, Gourdy P, Lenfant F (2013) In vivo dissection of the estrogen receptor alpha: uncoupling of its physiological effects and medical perspectives. Ann Endocrinol (Paris) 74:82-89

15. Vrtacnik P, Ostanek B, Mencej-Bedrac S, Marc J (2014) The many faces of estrogen signaling. Biochem Med (Zagreb) 24:329-342

16. Smith CL, O'malley BW (2004) Coregulator function: a key to understanding tissue specificity of selective receptor modulators. Endocr Rev 25:45-71
17. Brann DW, Zhang QG, Wang RM, Mahesh VB, Vadlamudi RK (2008) PELP1-a novel estrogen receptor-interacting protein. Mol Cell Endocrinol 290:2-7

18. Acconcia F, Kumar R (2006) Signaling regulation of genomic and nongenomic functions of estrogen receptors. Cancer Lett 238:1-14

19. Nair S, Vadlamudi RK (2007) Emerging significance of ERcoregulator PELP1/MNAR in cancer. Histol Histopathol 22:91-96

20. Chakravarty D, Nair SS, Santhamma B, Nair BC, Wang L, Bandyopadhyay A, Agyin JK, Brann D, Sun LZ, Yeh IT, Lee FY, Tekmal RR, Kumar R, Vadlamudi RK (2010) Extranuclear functions of ER impact invasive migration and metastasis by breast cancer cells. Cancer Res 70:4092-4101

21. Dang DN, Raj G, Sarode V, Molberg KH, Vadlamudi RK, Peng Y (2015) Significantly increased PELP1 protein expression in primary and metastatic triple-negative breast carcinoma: comparison with GATA3 expression and PELP1's potential role in triple-negative breast carcinoma. Hum Pathol 46:1829-1835

22. Roy S, Chakravarty D, Cortez V, De Mukhopadhyay K, Bandyopadhyay A, Ahn JM, Raj GV, Tekmal RR, Sun L, Vadlamudi RK (2012) Significance of PELP1 in ER-negative breast cancer metastasis. Mol Cancer Res 10:25-33

23. Stevis PE, Deecher DC, Suhadolnik L, Mallis LM, Frail DE (1999) Differential effects of estradiol and estradiol-BSA conjugates. Endocrinology 140:5455-5458

24. Zhang Y, Wang P, Shi M, Sasano H, Chan MS, Dai J, Guo L, Liu M, Wang X, Ma Y, Wang L (2014) The pattern of proline, glutamic acid, and leucine-rich protein 1 expression in Chinese women with primary breast cancer. Int J Biol Markers 29:e1-e7

25. Habashy HO, Powe DG, Rakha EA, Ball G, Macmillan RD, Green AR, Ellis IO (2010) The prognostic significance of PELP1 expression in invasive breast cancer with emphasis on the ER-positive luminal-like subtype. Breast Cancer Res Treat 120:603-612

26. Xu Y, Li Z, Jiang P, Wu G, Chen K, Zhang X, Li X (2015) The coexpression of MMP-9 and Tenascin-C is significantly associated with the progression and prognosis of pancreatic cancer. Diagn Pathol 10:211

27. Chen Y, Hong DY, Wang J, Ling-Hu J, Zhang YY, Pan D, Xu YN, Tao L, Luo H, Shen XC (2017) Baicalein, unlike 4hydroxytamoxifen but similar to G15, suppresses 17 betaestradiol-induced cell invasion, and matrix metalloproteinase- 9 expression and activation in MCF-7 human breast cancer cells. Oncol Lett 14:1823-1830

28. Nilsson UW, Dabrosin C (2006) Estradiol and tamoxifen regulate endostatin generation via matrix metalloproteinase activity in breast cancer in vivo. Cancer Res 66:4789-4794

29. Seeger H, Wallwiener D, Mueck AO (2006) Different effects of estradiol and various antiestrogens on TNF-alpha-induced changes of biochemical markers for growth and invasion of human breast cancer cells. Life Sci 78:1464-1468

30. Razandi M, Pedram A, Park ST, Levin ER (2003) Proximal events in signaling by plasma membrane estrogen receptors. J Biol Chem 278:2701-2712

31. Nilsson UW, Jonsson JA, Dabrosin C (2009) Tamoxifen decreases extracellular TGF-betal secreted from breast cancer cells-a posttranslational regulation involving matrix metalloproteinase activity. Exp Cell Res 315:1-9

32. Seeger H, Wallwiener D, Mueck AO (2008) Effects of estradiol and progestogens on tumor-necrosis factor-alpha-induced changes of biochemical markers for breast cancer growth and metastasis. Gynecol Endocrinol 24:576-579

33. Russo LA, Peano BJ, Trivedi SP, Cavalcanto TD, Olenchock BA, Caruso JA, Smolock AR, Vishnevsky O, Gardner RM (2009) Regulated expression of matrix metalloproteinases, inflammatory mediators, and endometrial matrix remodeling by 17 beta-estradiol in the immature rat uterus. Reprod Biol Endocrinol 7:124 
34. Cui H, Yuan J, Du X, Wang M, Yue L, Liu J (2015) Ethyl gallate suppresses proliferation and invasion in human breast cancer cells via Akt-NF-kappaB signaling. Oncol Rep 33:1284-1290

35. Ko HS, Lee HJ, Kim SH, Lee EO (2012) Piceatannol suppresses breast cancer cell invasion through the inhibition of MMP-9: involvement of PI3K/AKT and NF-kappaB pathways. J Agric Food Chem 60:4083-4089

36. Lee KS, Lee DH, Chun SY, Nam KS (2014) Metastatic potential in MDA-MB-231 human breast cancer cells is inhibited by proton beam irradiation via the Akt/nuclear factor-kappaB signaling pathway. Mol Med Rep 10:1007-1012
37. Vadlamudi RK, Wang RA, Mazumdar A, Kim Y, Shin J, Sahin A, Kumar R (2001) Molecular cloning and characterization of PELP1, a novel human coregulator of estrogen receptor alpha. J Biol Chem 276:38272-38279

38. Boonyaratanakornkit V (2011) Scaffolding proteins mediating membrane-initiated extra-nuclear actions of estrogen receptor. Steroids 76:877-884

Publisher's Note Springer Nature remains neutral with regard to jurisdictional claims in published maps and institutional affiliations. 